\title{
Mamíferos terrestres em um remanescente de Mata Atlântica, Paraná, Brasil
}

\author{
Márcia Regina Wolfart ${ }^{1 *}$ \\ Mateus Da Fré ${ }^{2}$ \\ Gustavo Borba de Miranda ${ }^{2}$ \\ Elaine Maria Lucas ${ }^{3}$ \\ ${ }^{1}$ AGROGER - Assessoria em Gestão Ambiental SS \\ Rua Rio de Janeiro, 35, Centro, CEP 85700-000, Barracão - PR, Brasil \\ ${ }^{2}$ Universidade do Oeste de Santa Catarina \\ Área das Ciências Biológicas e da Saúde, Laboratório de Biologia \\ Rua Oiapoc, 211, CEP 89900-000, São Miguel do Oeste - SC, Brasil \\ ${ }^{3}$ Universidade Comunitária da Região de Chapecó \\ Área de Ciências Exatas e Ambientais/Mestrado em Ciências Ambientais \\ Rua Senador Atílio Fontana, 591- E, CEP 89809-000, Chapecó - SC, Brasil \\ * Autor para correspondência \\ marciawolfart@yahoo.com.br
}

Submetido em 20/11/2012

Aceito para publicação em 02/10/2013

\section{Resumo}

O grau de ameaça e a importância ecológica dos mamíferos terrestres evidenciam a necessidade da constante realização de pesquisas com o intuito de acrescentar informações ao conhecimento atual sobre esse tema. Este estudo teve por objetivo fornecer uma lista de espécies de mamíferos terrestres em um remanescente de Mata Atlântica localizado no sudoeste do estado do Paraná. A riqueza de espécies e a frequência de ocorrência foram estudadas de abril a outubro de 2009, utilizando dois métodos: observação direta e registro de vestígios. Foram registrados 20 táxons distribuídos em sete ordens: Artiodactyla, Carnivora, Didelphimorphia, Lagomorpha, Primates, Rodentia e Xenarthra. Dentre estes, quatro táxons foram registrados tanto por observação direta quanto pelo registro de vestígios e os demais foram registrados somente por meio de vestígios. As espécies com ocorrência mais frequente foram Didelphis sp. (30,6\%) e Cerdocyon thous (25,6\%). Dos 20 táxons registrados, Leopardus pardalis, Leopardus tigrinus e Cuniculus paca constam como vulneráveis no Livro Vermelho da Fauna Ameaçada no Estado do Paraná. Apesar de pequena, a área de estudo deve auxiliar na disponibilidade de alimento e abrigo para a mastofauna, representando um importante elemento da paisagem regional.

Palavras-chave: Floresta ombrófila mista; Mamíferos; Mata Atlântica; Riqueza de espécies; Sudoeste do Paraná; Vestígios

\section{Abstract}

Terrestrial mammals in an Atlantic Forest remnant, Paraná, Brazil. The threat degree and the ecological importance of terrestrial mammals make clear the need for constantly conducting researches in order to add information to the current knowledge on this theme. This study aimed to provide a list of terrestrial mammal 
species in an Atlantic Forest remnant located in the Southwestern Paraná state, Brazil. Species richness and occurrence frequency were studied from April to October 2009 using two methods: direct observation and recording of traces. We registered 20 taxa distributed into 7 orders: Artiodactyla, Carnivora, Didelphimorphia, Lagomorpha, Primates, Rodentia, and Xenarthra. Among these, 4 taxa were registered either by direct observation or by recording of traces and the others were registered only through traces. The most frequently occurring species were Didelphis sp. (30.6\%) and Cerdocyon thous (25.6\%). Out of the 20 registered taxa, Leopardus pardalis, Leopardus tigrinus, and Cuniculus paca are listed as vulnerable in the Red Book of Threatened Fauna in Parana State. Although small, the study area may assist in the availability of food and shelter for the fauna of mammals, representing an important element of the regional landscape.

Key words: Atlantic Forest; Mammals; Ombrophilous mixed forest; Southwestern Paraná state; Species richness; Traces

\section{Introdução}

Estima-se que existam cerca de 5.416 espécies de mamíferos distribuídos no planeta (WILSON; REEDER, 2005). Destas, de acordo com Paglia et al. (2012), 701 são nativas no Brasil, das quais, 250 ocorrem na Mata Atlântica, sendo aproximadamente 22\% destas, endêmicas a este bioma (Reis et al., 2006). Na região sul do Brasil, percebe-se um incremento no conhecimento mastozoológico nos últimos anos, no entanto, ainda é uma região pouco estudada quanto à distribuição de sua mastofauna (CÁCERES et al., 2008). Para o estado do Paraná, a única lista, contendo 152 espécies, foi publicada por Lange e Jablonski (1981). Mikich et al. (2004), através do livro vermelho da fauna ameaçada no estado, acrescentaram informações sobre a ocorrência de 28 novas espécies, totalizando 180 mamíferos ocorrentes no estado, os quais representam cerca de $25 \%$ dos mamíferos conhecidos no Brasil.

A insuficiência de pesquisas sobre as espécies e suas distribuições geográficas é algo preocupante ao considerarmos a rápida velocidade de destruição dos ambientes naturais por ação antrópica (WILSON, 1997) e as consequências da redução de habitats à biota em função do isolamento genético das populações (PRADO et al., 2008). Além da redução de habitats, muitas espécies de mamíferos são apreciadas e perseguidas por caçadores e, em várias situações, estão sob constante ameaça pelo comércio ilegal de animais silvestres (MIKICH et al., 2004). O grau de ameaça e a importância ecológica dos mamíferos evidenciam a necessidade da realização de mais estudos em remanescentes florestais com o intuito de acrescentar novas informações ao conhecimento atual e contribuir na implantação de propostas que visem à conservação deste grupo.

A elaboração de listas de espécies constitui o primeiro passo para o monitoramento da fauna em determinada região (PIMENTA et al., 2005), além de contribuírem para o conhecimento dos habitats e para a avaliação do estado de conservação de espécies, sendo fundamentais para comparação com outras áreas e para orientar possíveis planos de manejo e de conservação da biodiversidade (PARDINI; UMETSU, 2006). Sem um conhecimento mínimo sobre quais espécies ocorrem em determinado local, é inviável desenvolver qualquer projeto ou implantar propostas de conservação (SANTOS, 2004). Neste sentido, este estudo teve como objetivo fornecer uma lista de espécies dos mamíferos terrestres ocorrentes em um remanescente de Mata Atlântica localizado no sudoeste do estado do Paraná visando contribuir sobre a lacuna do conhecimento mastofaunístico desta região e subsidiar a tomada de decisões sobre conservação.

\section{Material e Métodos}

O estudo foi desenvolvido em um remanescente de Mata Atlântica, com área aproximada de 220 ha, localizado no município de Bom Jesus do Sul, sudoeste do estado do Paraná (2606'37's; 5333'40”'O; Figura 1). Dos 220 ha, 46,7 ha estão registrados como Reserva Particular do Patrimônio Natural, denominada RPPN Carlos Valdir Maran. A vegetação da região é constituída por Floresta Ombrófila Mista, pertencente ao domínio do bioma Mata Atlântica (IBGE, 2012). O rio Capanema, pertencente à bacia hidrográfica do rio Iguaçu, atravessa 
a área de estudo e, em áreas antropizadas, apresenta mata ciliar constituída de vegetação em estágios iniciais ou intermediários de sucessão ou plantios de pastagem e Pinus sp. Já em áreas de mais difícil acesso e menor interferência humana, a mata ciliar é constituída de vegetação mais preservada.

O inventário da mastofauna foi realizado de abril a outubro de 2009, utilizando-se dois métodos: observação direta e registro de vestígios em parcelas de areia e fora destas. O método de visualização direta foi empregado durante três dias não consecutivos por mês, através de caminhadas lentas e em silêncio nas margens do rio, em estradas e na borda e interior da mata totalizando um esforço amostral de $35,7 \mathrm{~km}$. As observações eram realizadas procurando contemplar todos os ângulos possíveis e, desta forma, registrar mamíferos em suas atividades diurnas ou por meio de vestígios como pegadas e fezes.

Para o registro de vestígios foram instalados dois conjuntos de parcelas de areia no interior da mata. Cada conjunto $(\mathrm{C} 1$ e $\mathrm{C} 2)$ consistiu em três linhas paralelas com dez armadilhas de pegadas cada. O intervalo entre as linhas foi de $50 \mathrm{~m}$ e entre as armadilhas, $10 \mathrm{~m}$, totalizando 30 armadilhas por conjunto (Figura 1). As parcelas apresentavam três centímetros de profundidade, compreendendo uma área de $50 \mathrm{~cm}$ x $50 \mathrm{~cm}$ preenchidas com areia fina (PARDINI et al., 2004).

A coleta de dados nas parcelas de areia foi realizada durante três dias consecutivos por mês, totalizando esforço amostral de 20 dias, sendo que nos dias anteriores às coletas, fazia-se a descompactação da areia das armadilhas que posteriormente eram umedecidas com o auxílio de um borrifador e iscadas com banana, laranja, bacon e pasta de amendoim. As parcelas eram iscadas diariamente e os rastros deixados pelos mamíferos eram medidos, fotografados em escala e, na maioria das vezes, eram produzidos contramoldes de gesso para auxiliar na identificação (CARVALHO-JÚNIOR; LUZ, 2008). A identificação dos rastros foi baseada em Becker e Dalponte (1991);

FIGURA 1: Localização geográfica mostrando a distribuição das linhas de parcelas de areia (C1 e C2) na área de estudo no município de Bom Jesus do Sul, sudoeste do Paraná.

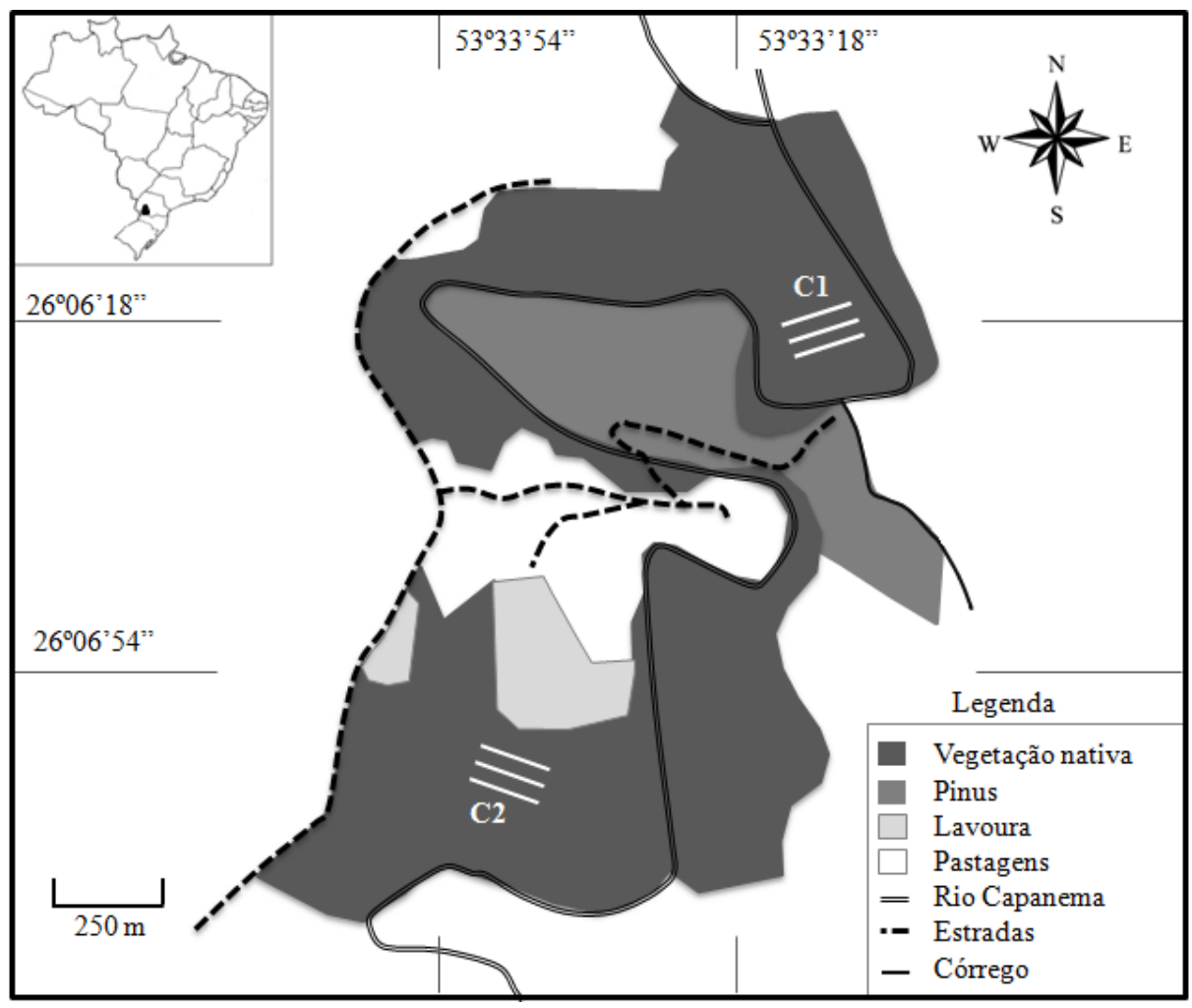


Oliveira e Cassaro (2006); Borges e Tomás (2008); Carvalho-Júnior e Luz (2008) e Moro-Rios et al. (2008) e a nomenclatura seguiu Reis et al. (2006). Cada vestígio ou visualização foi considerado um registro, exceto quando as pegadas formavam trilhas, neste caso, cada trilha era considerada um registro. $\mathrm{O}$ número de registros de cada espécie foi utilizado como indicativo de sua frequência de ocorrência na área de estudo. O número total de registros em cada cojunto foi comparado através do teste estatístico Kuskal-Wallis, para isto utilizou-se o pacote estatístico BioEstat 5.3.

\section{Resultados}

Foram registrados 20 táxons de mamíferos terrestres distribuídos em sete ordens e 16 famílias (Tabela 1), representando $11 \%$ da riqueza de mamíferos conhecida para o Estado do Paraná $(\mathrm{n}=180$ espécies; Mikich et al., 2004). Carnivora e Rodentia foram as ordens com maior número de espécies $(n=7)$, seguidas da ordem Didelphimorphia $(n=2)$. As ordens Xenarthra, Primates, Lagomorpha e Artiodactyla foram representadas por apenas uma espécie cada (Tabela 1).

TABELA 1: Táxons, nome comum, forma de registro (FR): pegadas (P), fezes (F), visualização (V), espinhos (E), número de registro nas parcelas de areia (NRP), número de registro fora das parcelas de areia (NRFP), total de registros por táxon (RT) e frequência de ocorrência (FO) de mamíferos terrestres registrados em um remanescente de Mata Atlântica, no município de Bom Jesus do Sul, sudoeste do estado do Paraná no período de abril a outubro de 2009.

\begin{tabular}{|c|c|c|c|c|c|c|}
\hline TÁXONS & NOME COMUM & FR & NRP & NRFP & RT & FO \\
\hline \multicolumn{7}{|l|}{ ARTIODACTYLA } \\
\hline Mazama sp. & Veado & $\mathrm{P}$ & 0 & 1 & 1 & $0,2 \%$ \\
\hline \multicolumn{7}{|l|}{ CARNIVORA } \\
\hline Cerdocyon thous & Graxaim & $\mathrm{P} / \mathrm{F}$ & 150 & 8 & 158 & $25,6 \%$ \\
\hline Leopardus pardalis & Jaguatirica & $\mathrm{P}$ & 0 & 9 & 9 & $1,5 \%$ \\
\hline Leopardus tigrinus & Gato-do-mato-pequeno & $\mathrm{P}$ & 1 & 2 & 3 & $0,5 \%$ \\
\hline Puma yagouaroundi & Gato mourisco & $\mathrm{P}$ & 1 & 0 & 1 & $0,2 \%$ \\
\hline Eira barbara & Irara & $\mathrm{P}$ & 3 & 0 & 3 & $0,5 \%$ \\
\hline Procyon cancrivorus & Mão-pelada & $\mathrm{P} / \mathrm{V}$ & 19 & 44 & 63 & $10,2 \%$ \\
\hline Nasua nasua & Coati & $\mathrm{P} / \mathrm{V}$ & 0 & 3 & 3 & $0,5 \%$ \\
\hline \multicolumn{7}{|l|}{ DIDELPHIMORPHIA } \\
\hline Didelphis sp. & Gambá & $\mathrm{P}$ & 181 & 8 & 189 & $30,6 \%$ \\
\hline Subfamília Caluromyinae & Cuíca & $\mathrm{P}$ & 0 & 2 & 2 & $0,3 \%$ \\
\hline \multicolumn{7}{|l|}{ LAGOMORPHA } \\
\hline Sylvilagus brasiliensis & Tapiti & $\mathrm{P} / \mathrm{F}$ & 5 & 1 & 6 & $1,0 \%$ \\
\hline \multicolumn{7}{|l|}{ PRIMATES } \\
\hline Cebus nigritus & Macaco-prego & $\mathrm{P}$ & 0 & 1 & 1 & $0,2 \%$ \\
\hline \multicolumn{7}{|l|}{ RODENTIA } \\
\hline Cavia sp. & Preá & $\mathrm{P} / \mathrm{V}$ & 0 & 4 & 4 & $0,6 \%$ \\
\hline Cuniculus paca & Paca & $\mathrm{P}$ & 0 & 54 & 54 & $8,8 \%$ \\
\hline Dasyprocta azarae & Cotia & $\mathrm{P}$ & 8 & 16 & 24 & $3,9 \%$ \\
\hline Família Cricetidae & & $\mathrm{P}$ & 0 & 59 & 59 & $9,6 \%$ \\
\hline Guerlinguetus ingrami & Serelepe & $\mathrm{P} / \mathrm{V}$ & 5 & 3 & 8 & $1,3 \%$ \\
\hline Myocastor coypus & Ratão-do-banhado & $\mathrm{P}$ & 0 & 2 & 2 & $0,3 \%$ \\
\hline Coendou sp. & Ouriço caixeiro & E & 0 & 1 & 1 & $0,2 \%$ \\
\hline \multicolumn{7}{|l|}{ XENARTHRA } \\
\hline \multirow{2}{*}{ Dasypus sp. } & Tatu & $\mathrm{P}$ & 22 & 4 & 26 & $4,2 \%$ \\
\hline & & TOTAL & 395 & 222 & 617 & \\
\hline
\end{tabular}


Entre os vinte táxons ocorrentes na área de estudo, dez foram registrados somente fora das armadilhas de pegadas: Mazama sp. (Rafinesque, 1817), Leopardus pardalis (Linnaeus, 1758), Cebus nigritus (Goldfuss, 1809), Cavia sp. (Pallas, 1766), Cuniculus paca (Linnaeus, 1758), Myocastor coypus (Molina, 1782), Nasua nasua (Linnaeus, 1766), Coendou sp. (Lacépède, 1809), um roedor da família Cricetidae e uma espécie da subfamília Caluromyinae; dois foram registrados somente por meio de pegadas deixadas nas armadilhas: Puma yagouaroundi (Lacépède, 1809) e Eira barbara (Linnaeus, 1758) e os outros oito táxons: Cerdocyon thous (Linnaeus, 1766), Leopardus tigrinus (Shreber, 1775), Procyon cancrivorus (Cuvier, 1798), Didelphis sp. (Linnaeus, 1758), Sylvilagus brasiliensis (Linnaeus, 1758), Dazyprocta azarae (Lichtenstein, 1823), Guerlinguetus ingrami (Thomas, 1901) e Dasypus sp. (Linnaeus, 1758) foram registrados tanto nas parcelas de areia como por meio de visualizações e de vestígios encontrados fora destas (Tabela 1).

O método de registro de pegadas mostrou-se mais eficiente para o levantamento de mamíferos na área de estudo, possibilitando identificar 19 espécies (Tabela 1). A utilização de rastros para identificação dos mamíferos é uma alternativa simples, por serem informativos e facilmente encontrados (Becker; Dalponte, 1991). Além das pegadas, foram encontrados espinhos de Coendou sp. (ouriço caixeiro) próximo da estrada de acesso à sede da propriedade. Houve também três registros de fezes: dois de Cerdocyon thous (graxaim) e um de Sylvilagus brasiliensis (tapiti), as quais corroboraram com as descritas por Borges e Tomás (2008). Procyon cancrivorus (mão-pelada), Nasua nasua (coati), Cavia sp. (preá) e Guerlinguetus sp. (serelepe), foram registradas através de pegadas e visualização (Tabela 1).

Os táxons mais frequentemente registrados foram Didelphis sp., Cerdocyon thous, Procyon cancrivorus, o rato d'água pertencente à família Cricetidae e Cuniculus paca, que apresentaram frequências de ocorrência de $30,6 \%, 25,6 \%, 10,2 \%, 9,6 \%$ e $8,8 \%$, respectivamente. Já as menos frequentes foram Dasypus sp. com 4,2\% do total dos registros, Dasyprocta azarae com 3,9\%,
Leopardus pardalis (1,5\%), Guerlinguetus ingrami (1,3\%), Sylvilagus brasiliensis (1,0\%), Cavia sp. (0,6\%), Eira barbara, Leopardus tigrinus e Nasua nasua com $0,5 \%$ dos registros cada, uma espécie de cuíca da subfamília Caluromyinae e Myocastor coypus com $0,3 \%$ cada e Mazama sp., Puma yagouaroundi, Cebus nigritus e Coendou sp. com 0,2\% cada (Tabela 1).

Com relação à comparação entre os números totais de registros em cada conjunto e áreas de entorno, o teste estatístico mostrou uma diferença significativa $(\mathrm{H}=3,9224 ; P=0,0476)$ entre os mesmos, tendo o Conjunto 2 um maior número de registros (393), quando comparado ao Conjunto 1 (224, Tabela 2). No entanto, alguns táxons foram registrados exclusivamente no Conjunto 1 e áreas de entorno (Leopardus tigrinus, uma espécie da subfamília Caluromyinae, Myocastor coypus e uma espécie de Mazama sp.) e outros táxons foram registrados apenas no conjunto 2 e áreas de entorno (Nasua nasua, Puma yagouaroundi, Cebus nigritus e Coendou sp.). Esta diferença no uso de habitat e no número de registros por táxon, principalmente para Didelphis sp. e Cerdocyon thous entre os locais de amostragem, pode estar associada às interferências antrópicas, tendo em vista que as áreas de entorno do Conjunto 1 são frequentemente utilizadas por moradores para lazer e pesca e a matriz é constituída por plantio de Pinus sp. Também há pastagens nas áreas próximas, nas quais é comum a presença de gado em locais de fácil acesso ao Rio Capanema. Já as áreas de entorno do Conjunto 2 são de difícil acesso o que provavelmente as mantém melhor preservadas. 
TABELA 2: Táxons, número de registro de mamíferos terrestres no conjunto 1 e áreas de entorno (NRC1) e número de registros no conjunto $2 \mathrm{e}$ áreas de entorno (NRC2) em um remanescente de Mata Atlântica, no município de Bom Jesus do Sul, sudoeste do estado do Paraná no período de abril a outubro de 2009.

\begin{tabular}{lcc}
\hline Táxons & NRC1 & NRC2 \\
\hline Didelphis sp. & 116 & 73 \\
Cerdocyon thous & 16 & 142 \\
Procyon cancrivorus & 15 & 48 \\
Família Cricetidae & 12 & 47 \\
Cuniculus paca & 19 & 35 \\
Dasypus sp. & 18 & 8 \\
Dasyprocta azarae & 10 & 14 \\
Leopardus pardalis & 4 & 5 \\
Guerlinguetus ingrami & 2 & 6 \\
Sylvilagus brasiliensis & 2 & 4 \\
Cavia sp. & 1 & 3 \\
Eira barbara & 1 & 2 \\
Leopardus tigrinus & 3 & 0 \\
Nasua nasua & 0 & 3 \\
Subfamília Caluromyinae & 2 & 0 \\
Myocastor coypus & 2 & 0 \\
Mazama sp. & 1 & 0 \\
Puma yagouaroundi & 0 & 1 \\
Cebus nigritus & 0 & 1 \\
Coendou sp. & 0 & 1 \\
\hline TOTAL & 224 & 393 \\
\hline
\end{tabular}

\section{Discussão}

A alta frequência de registros em parcelas de areia, principalmente de Didelphis sp., Cerdocyon thous e Procyon cancrivorus pode estar relacionada às limitações do método utilizado ou seja, pela falta de independência entre as parcelas de areia, uma vez que um único indivíduo pode ter sido registrado em várias parcelas consecutivas, superestimando a frequência das espécies nas áreas amostradas. De acordo com Scoss e De-Marco (2000), este problema poderia ser resolvido aumentando a distância entre as parcelas. Entretanto, os marsupiais são bastante comuns em toda sua área de distribuição, demonstrando eficiência adaptativa aos mais variados hábitats, até mesmo a centros urbanos (ROSSI et al., 2006).

Na região sul do Brasil, ocorrem duas espécies deste gênero: D. albiventris (gambá-de-orelha-branca) e
D. aurita (gambá-de-orelha-preta) e a distribuição destes gambás é complexa e entrelaçada, podendo ocorrer em simpatria em algumas localidades (EMMONS; FEER, 1999; CÁCERES et al., 2008). Por isso, e pelo fato destes marsupiais diferenciarem-se apenas pela coloração do pavilhão auditivo, não havendo diferença entre suas pegadas (SILVA, 1994), foi considerado neste trabalho o gênero Didelphis. A presença de poucos predadores de maior porte em pequenos fragmentos pode levar a um aumento da abundância de espécies generalistas como o gambá (FONSECA; ROBINSON, 1990). Isto pode estar ocorrendo na área de estudo já que, mesmo com a ordem Carnivora apresentando um dos maiores números de indivíduos, a frequência de registros de espécies generalistas como Didelphis sp. foi bem maior quando comparada com a frequência de registros de carnívoros como Puma yagouaroundi, Leopardus pardalis, Leopardus tigrinus e Eira barbara (Tabela 1).

O número de táxons observados neste estudo foi aproximado ao número registrado em outros remanescentes de Mata Atlântica, entretanto, há de se considerar as diferenças em relação ao tamanho da área, esforço amostral e métodos utilizados (Tabela 3). Negrão e Valladares-Pádua (2006) registraram 18 espécies em sete áreas dentro da Reserva Florestal do Moro Grande, em São Paulo (Tabela 3). Destas, Sciurus aestuans (esquilo), Didelphis aurita (gambá) e Sylvilagus brasiliensis (tapiti) foram as mais registradas e estavam presentes em quase todas as áreas amostradas (NEGRÃO; VALLADARES-PÁDUA, 2006). Kasper et al. (2007), em pesquisa realizada no Parque Estadual do Turvo, no Rio Grande do Sul, registraram 29 espécies, das quais, Dazyprocta azarae (cotia) e Sylvilagus brasiliensis foram as espécies com maior número de registros (Tabela 3). Em outra pesquisa realizada em um fragmento de Mata Atlântica em Minas Gerais, Prado et al. (2008) registraram 23 espécies de mamíferos terrestres sendo que o maior número de registros foi da espécie exótica Canis lupus familiaris seguida por Cerdocyon thous, um dos táxons silvestres considerado oportunista em relação à dieta, sobrevivendo em áreas degradadas e antrópicas (MOTTA-JÚNIOR et al., 1994; FACURE; MONTEIRO-FILHO, 1996). Em dois pequenos fragmentos de Mata Atlântica com 1,0 e 1,2 ha localizados na zona sul do município de Londrina, 
TABELA 3: Comparação entre o número de táxons registrados neste trabalho e número de táxons registrados em outras pesquisas realizadas em remanescentes de Mata Atlântica no Brasil.

\begin{tabular}{|c|c|c|c|c|}
\hline Táxon & Área (ha) & Metodologia & Esforço & Autores \\
\hline 20 & 220 & \begin{tabular}{|l|} 
Identificação de rastros \\
Visualizações \\
\end{tabular} & $\begin{array}{c}20 \text { dias } \\
35,7 \mathrm{~km} \\
\end{array}$ & Presente trabalho \\
\hline 18 & 10.870 & $\begin{array}{l}\text { Identificação de rastros } \\
\text { Visualizações }\end{array}$ & $\begin{array}{c}\text { sem estimativas } \\
241 \mathrm{~km} \text { (transectos) }\end{array}$ & Negrão; Valladares-Pádua (2006) \\
\hline 29 & 17.491 & \begin{tabular}{|l} 
Armadilhas fotográficas \\
Identificação de rastros \\
Visualizações \\
\end{tabular} & $\begin{array}{c}960 \text { por noite } \\
128 \mathrm{~km} \\
\text { sem estimativas }\end{array}$ & Kasper et al. (2007) \\
\hline 23 & 384,5 & $\begin{array}{l}\text { Armadilhas fotográficas } \\
\text { Armadilhas Tomahawk } \\
\text { Identificação de sinais } \\
\text { acústicos, rastros, fezes, } \\
\text { pelos e carcaças }\end{array}$ & $\begin{array}{c}198 \text { dias } \\
21 \text { noites } \\
\\
\text { sem estimativas } \\
\end{array}$ & Prado et al. (2008) \\
\hline 16 & 1,0 e 1,2 & $\begin{array}{l}\text { Armadilhas fotográficas } \\
\text { Identificação de rastros } \\
\text { Visualizações } \\
\text { Entrevistas }\end{array}$ & $\begin{array}{c}40 \text { dias } \\
20 \text { dias } \\
40 \text { dias } \\
\text { não padronizadas }\end{array}$ & Lopes et al. (2009) \\
\hline
\end{tabular}

Estado do Paraná, Lopes et al. (2009) registraram 16 espécies (Tabela 3) contemplando importantes carnívoros os quais levaram os autores a concluir que, pelo fato destes pequenos remanescentes estarem ainda rodeados por outros maiores, incluindo o Parque Estadual Mata dos Godoy, com 346 ha, ainda apresentam pelo menos condições mínimas para o trânsito, mesmo que temporário de Eira barbara, Leopardus pardalis e Puma concolor.

Duas espécies registradas neste estudo, Leopardus pardalis e Leopardus tigrinus constam como vulneráveis no Livro Vermelho da Fauna Ameaçada no Estado do Paraná (MIKICH; BÉRNILS, 2004) e no Livro Vermelho da Fauna Brasileira Ameaçada de Extinção (CHIARELLO et al., 2008). Mesmo ausentes no Livro Vermelho da Fauna Brasileira, Cuniculus paca e uma espécie do gênero Mazama sp., constam como espécies vulneráveis à extinção no Estado do Paraná (MIKICH; BÉRNILS, 2004). Porém, ainda não foi possível confirmar qual espécie do gênero Mazama ocorre na área.

Segundo Pitman e Oliveira (2002), a presença de mamíferos de topo de cadeia alimentar, os quais podem influenciar diretamente a dinâmica do ecossistema em que vivem, como Eira barbara, Leopardus pardalis, Leopardus tigrinus e Puma yagouaroundi, registrados no presente trabalho, pode ser um indicativo de que o fragmento estudado auxilia na manutenção destes táxons na escala regional. Porém, são necessários estudos populacionais que avaliem a dinâmica espacial das espécies, incluindo análises de variabilidade genética e dos fatores ecológicos que contribuem para a manutenção destes táxons em médio ou longo prazo.

De maneira geral, este estudo reforça o padrão encontrado em uma grande parte das pesquisas realizadas com mamíferos em remanescentes de Mata Atlântica (Tabela 3), com maior frequência de registros de espécies generalistas, principalmente roedores e carnívoros de pequeno porte. Como a região de estudo é caracterizada por fragmentos pequenos e isolados (RIBEIRO et al., 2009), com exceção das áreas contínuas de floresta do lado Argentino e do Parque Nacional do Iguaçu, os mamíferos de grande porte estão provavelmente extintos regionalmente. Desta forma, algumas espécies como o graxaim, o mão-pelada e a paca devem ser especialmente importantes do ponto de vista da conservação nesta região, pois provavelmente são os maiores responsáveis pela dispersão de sementes grandes, uma vez que esses animais se alimentam de sementes maiores e se deslocam 
amplamente entre os fragmentos (PIZO, 2012). A falta de dispersores de sementes grandes é comum em paisagens fragmentadas e ameaça o futuro das comunidades vegetais em todo o mundo (PIZO, 2012).

No entanto, mesmo com a presença de carnívoros dispersores de sementes grandes e, mesmo com a frequência de ocorrência relativamente alta registrada para Cuniculus paca $(8,8 \%)$, considerada uma importante dispersora e predadora de sementes e plântulas (DIRZO; MIRANDA, 1990) e constantemente ameaçada pela caça e destruição das matas ciliares (MIKICH; BÉRNILS, 2004), o registro único de Mazama sp., Puma yagouaroundi, Cebus nigritus e Coendou sp. pode indicar que estas espécies são raras na área de estudo.

A raridade de determinadas espécies na área, associada a relatos da ação de caçadores, a presença de cães domésticos, bovinos e o plantio de Pinus sp. em áreas de entorno, sugere uma constante pressão antrópica neste remanescente de Mata Atlântica. Mesmo não sofrendo redução significativa de área florestal, as interferências antrópicas podem ocasionar deterioração ambiental e consequente defaunação local. Canale et al. (2012) enfatizam que a extinção de espécies de mamíferos na Mata Atlântica pode ser muito mais uma consequência de efeitos sinérgicos (impactos antropogênicos diversos, incluindo a caça) do que somente perda de área, não existindo uma relação direta de espécie-área apesar de muitas espécies de Mammalia necessitarem de áreas relativamente grandes para a sobrevivência.

Desta forma, a maior importância da área de estudo está relacionada ao contexto da paisagem, pois esta deve auxiliar nas necessidades de abrigo, alimentação e reprodução das populações de mamíferos da região. Porém, para minimizar os impactos negativos existentes, recomenda-se o desenvolvimento de projetos de educação socioambiental envolvendo as comunidades do entorno, visando a sensibilização sobre a importância da conservação das espécies e da redução das atividades com potencial efeito negativo sobre as populações. Além disso, é importante a realização de estudos sobre a estrutura e a dinâmica espacial dos táxons registrados, com foco nas espécies dispersoras de sementes, as quais contribuem para a regeneração natural das florestas permitindo a definição de políticas públicas adequadas à conservação da biodiversidade regional.

Tendo em vista que o remanescente estudado possui aproximadamente 220 ha, dos quais apenas 46,7 ha estão registrados como Reserva Particular do Patrimônio Natural, seria viável proteger legalmente todo o remanescente e realizar o plano de manejo para a área. A continuação dos estudos acrescentando outras metodologias, como o uso de armadilhas fotográficas, poderia refinar a identidade taxonômica de alguns táxons registrados (Mazama sp., Didelphis sp., Coendou sp, Dasypus sp. entre outros) e fornecer informações sobre o tamanho populacional das espécies. A utilização de armadilhas live traps e pitfall traps poderá fornecer informações sobre a composição de roedores de pequeno porte, e a inclusão da ordem Chiroptera aprofundaria ainda mais o conhecimento sobre a diversidade de mamíferos da área estudada. A utilização de técnicas moleculares, considerando a conservação da diversidade genética nas populações, poderia determinar quais espécies precisam de maior atenção na determinação de estratégias regionais de conservação (PEREZSWEENEY et al., 2004), possibilitando traçar metas para a conservação da área de forma mais efetiva.

\section{Agradecimentos}

Aos proprietários da área estudada: Dona Filomena Maran e seus filhos, em especial Oilson Maran e Marli Maran por permitirem que este estudo fosse realizado. À Dra . Vanessa Barbisan Fortes pelo auxílio na identificação de pegadas. Ao Dr. Adriano Dias de Oliveira pelas sugestões no trabalho. A Roberta Cristina Della Méa, Denise Brancher e Gilberto Monteiro Garcia pelo auxílio em campo.

\section{Referências}

BECKER, M.; DALPONTE, J. C. Rastros de mamíferos silvestres brasileiros: um guia de campo. Brasília: UnB, 1991. 180 p.

BORGES, P. A. L.; TOMÁS, W. M. Guia de rastros e outros vestígios de mamíferos do pantanal. Corumbá: Embrapa Pantanal, 2008. 139 p.

CÁCERES, N. C.; CHEREM, J. J.; GRAIPEL, M. E. Distribuição geográfica de mamíferos terrestres na Região Sul do Brasil. Ciência \& Ambiente, Santa Maria, n. 35, p. 167-180, 2008. 
CANALE, G. R.; PERES, C. A.; GUIDORIZZI, C. E.; FERREIRAGATTO, C. A.; KIERULFF, C. M. Pervasive defaunation of forest remmants in a tropical biodiversity hotspot. Plos One, Cambridge, v. 7, n. 8, p. 1-9, 2012.

CARVAlHO-JÚNIOR, O.; LUZ, N. C. Pegadas: Série Boas Práticas. Belém: EDUFPA, 2008. 64 p.

CHIAREllO, A. G.; AGUIAR, L. M. S.; CERQUEIRA, R.; MELO, F. R.; RODRIGUES, F. H. G.; SILVA, V. M. F. Mamíferos ameaçados de extinção no Brasil. In: MACHADO, A. B. M.; DRUMMOND G. M.; PAGLIA, A. P. (Ed.). Livro vermelho da fauna brasileira ameaçada de extinção. Brasília: MMA; Belo Horizonte: Fundação Biodiversitas, 2008. p. 681-874.

COSTA, L. P.; LEITE, Y. L. R.; MENDES, S. L.; DITCHFIELD, A. D. Mammal conservation in Brazil. Conservation Biology, San Francisco, v. 19, n. 3, p. 672-679, 2005.

DIRZO, R.; MIRANDA, A. Contemporary neotropical defaunation and forest structure, function and diversity: a sequel to John Terborgh. Conservation Biology, San Francisco, v. 4, n. 4, p. 444447, 1990 .

EMMONS, L. H.; FEER, F. Neotropical rainforest mammals: a field guide. Chicago: University of Chicago, 1999. 396 p.

FACURE, K. G.; MONTEIRO-FILHO, E. L. A. Feeding habits of the crab-eating fox, Cerdocyon thous (Carnivora, Canidae), in a suburban area of southeastern Brazil. Mammalia, Paris, v. 60, n. 1, p. 147-149, 1996.

FONSECA, G. A. B.; ROBINSON, J. G. Forest size and structure: competitive and predatory effects on small mammal communities. Biological Conservation, Boston, v. 53, n. 4, p 265-294, 1990.

IBGE - INSTITUTO BRASILEIRO DE GEOGRAFIA E ESTATÍSTICA. Manual técnico da vegetação brasileira: Série Manuais Técnicos em Geociências. no ${ }^{-1}$. 2. ed. Rio de Janeiro: IBGE, 2012. $271 \mathrm{p}$.

KASPER, C. B.; MAZIM, F. D.; SOARES, J. B. G.; OLIVEIRA, T. G.; FABIÁN, M. E. Composição e abundância relativa dos mamíferos de médio e grande porte no Parque Estadual do Turvo, Rio Grande do Sul, Brasil. Revista Brasileira de Zoologia, Curitiba, v. 24, n. 4, p. 1087-1099, 2007.

LANGE, R. B.; JABLONSKI, E. F. Lista prévia dos Mammalia do Estado do Paraná. Revista Estudos de Biologia, Curitiba, n. 6, p. $1-35,1981$.

LOPES, P. A. M.; ORSI, M. L.; MARIOTO, A. C. Levantamento da fauna de mamíferos em dois fragmentos da zona sul do município de Londrina, Estado do Paraná. In: CONGRESSO DE ECOLOGIA DO BRASIL, 9, 2009, São Lourenço. Anais... São Lourenço, 2009. Versão eletrônica.

MIKICH, S. B.; BÉRNILS, R. S. Livro vermelho da fauna ameaçada no estado do Paraná. Curitiba: Instituto Ambiental do Paraná, 2004. 763 p.

MIKICH, S. B.; BÉRNILS, R. S.; PIZZI, P. A. Fauna ameaçada no Paraná: uma introdução. In: MIKICH, S. B.; BÉRNILS, R. S. (Ed.). Livro vermelho da fauna ameaçada no estado do Paraná. Curitiba: Instituto Ambiental do Paraná, 2004. p. 3.

MORO-RIOS, R. F.; SILVA-PEREIRA, J. E.; SILVA, P. W.; MOURA-BRITTO, M. PATROCÍNIO, D. N. M. Manual de rastros da fauna paranaense. Curitiba: Instituto Ambiental do Paraná, 2008. 70 p.
MOTTA-JÚNIOR, J. C.; LOMBARDI, J. A.; TALAMONI, S. A. Notes on crab-eating fox (Dusicyonthous) seed dispersal and food habits in southeastern Brazil. Mammalia, Paris, v. 58, p. 156-159, 1994.

NEGRÃO, M. F. F.; VALLADARES-PÁDUA, C. Registro de mamíferos de maior porte na Reserva Florestal do Morro Grande, São Paulo. Biota Neotropica, Campinas, v. 6, n. 2, p. 1-13, 2006.

OLIVEIRA, T. G.; CASSARO, K. Guia de campo dos felinos do Brasil. São Paulo: Instituto Pró-Carnívoros; Fundação Parque Zoológico de São Paulo, Sociedade de Zoológicos do Brasil, PróVida Brasil, 2006. 80 p.

PAGLIA, A. P.; FONSECA, G. DA; RYLANDS, A.; HERRMANN, G.; AGUIAR, L.; CHIARELLO, A. G.; LEITE, Y.; COSTA, L.; SICILIANO, S.; KIERULFF, M.; MENDES, S.; TAVARES, V.; MITTERMEIER, R.; PATTON, J. Lista anotada dos mamíferos do Brasil. 2. ed. Ocasional papers in conservation biology. Arlington: Conservation International, 2012. 76 p.

PARDINI, R.; DITT, E. H.; CULLEN-JUNIOR, L.; BASSI, C.; RUDRAN, R. Levantamento rápido de mamíferos terrestres de médio e grande porte. In: CULLEN-JÚNIOR, L.; RUDRAN, R.; VALLADARES-PÁDUA, C. (Ed.). Métodos de estudos em Biologia da Conservação e manejo da vida silvestre. Curitiba: Editora da UFPR \& Fundação O Boticário de Proteção à Natureza, 2004. p. 181-201.

PARDINI, R.; UMETSU, F. Pequenos mamíferos não-voadores da Reserva Florestal do Morro Grande: distribuição das espécies e da diversidade em uma área de Mata Atlântica. Biota Neotropica, Campinas, v. 6, n. 2, p. 1-22, 2006.

PEREZ-SWEENEY, B.; RODRIGUES, F. P.; MELNICK, D. J. Metodologias moleculares utilizadas em genética da conservação. In: CULLEN-JÚNIOR, L.; RUDRAN, R.; VALLADARESPÁDUA, C. (Ed.). Métodos de estudos em Biologia da Conservação e manejo da vida silvestre. Curitiba: Editora da UFPR \& Fundação O Boticário de Proteção à Natureza, 2004. p. 343-380.

PIMENTA, B. V. S.; HADDAD, C. F. B.; NASCIMENTO, L. B.; CRUZ, C. A. G.; POMBAL-JÚNIOR, J. P. Comment on "status and trends of amphibian declines and extinctions worldwide". Science, New York, v. 309, n. 5743, p. 1999, 2005.

PITMAN, M. R. P. L.; OLIVEIRA, T. G. Por que promover a conservação de carnívoros? In: PITMAN, M. R. P. L.; OLIVEIRA, T. G.; PAULA, R. C.; INDRUSIAK, C. (Ed.). Manual de identificação, prevenção e controle de predação por carnívoros. Brasília: Edições IBAMA, 2002. p. 21-23.

PIZO, M. A. O movimento dos animais frugívoros e das sementes em paisagens fragmentadas. In: DEL CLARO, K.; TOREZANSILINGARDO, H. M. (Org.). Ecologia das interações plantasanimais: uma abordagem ecológico-evolutiva. Rio de Janeiro: Technical Books Editora, 2012. p. 141-154.

PRADO, M. R.; ROCHA, E. C.; GIUDICE, G. M. L. Mamíferos de médio e grande porte em um fragmento de Mata Atlântica, Minas Gerais, Brasil. Revista Árvore, Viçosa, v. 32, n. 4, p. 741-749, 2008.

REIS, N. R.; SHIBATTA, O. A.; PERACCHI, A. L.; PEDRO, W. A.; LIMA, I. P. Mamíferos do Brasil. Londrina: Universidade de Londrina, 2006. 437 p. 